\title{
Preoperative Computed Tomography-Derived Bone Densities in Hounsfield Units at Implant Sites Acquired Primary Stability
}

\author{
Kyou Hiasa, Yasuhiko Abe, Yohei Okazaki, Keisuke Nogami, \\ Wataru Mizumachi, and Yasumasa Akagawa
}

Division of Cervico-Gnathostomatology, Department of Advanced Prosthodontics, Graduate School of Biomedical Sciences,
Hiroshima University, Hiroshima 734-8553, Japan

Correspondence should be addressed to Yasuhiko Abe, abey@hiroshima-u.ac.jp

Received 1 September 2011; Accepted 27 September 2011

Academic Editor: M. I. Ryder

Copyright (๑) 2011 Kyou Hiasa et al. This is an open access article distributed under the Creative Commons Attribution License, which permits unrestricted use, distribution, and reproduction in any medium, provided the original work is properly cited.

\begin{abstract}
The purpose of this study was to evaluate preoperative CT-derived bone densities in Hounsfield units (HU) at implant sites that acquired primary stability, and to compare these values to the optimal bone densities proposed in the literature. Fiftyone patients, 18 males (37 implant sites) and 33 females (67 implant sites) between 2003 and 2010 were assessed. CT data for different jaw sections, regions, and operating procedures were compared using the Kruskal-Wallis test and Scheffe's test for multiple comparisons $(P<0.05)$. The mean bone density in the maxilla was significantly lower than that in the mandible $(P<0.05)$; the mean bone densities in the 4 jaw regions decreased in the following order: anterior mandible $>$ anterior maxilla $>$ posterior mandible > posterior maxilla. The bone densities assessed by HU fell into the range of optimal bone densities associated with acquired primary implant stability proposed in the literature.
\end{abstract}

\section{Introduction}

Oral implant success is mainly influenced by bone quality and bone quantity. Bone quality is an important factor for achieving primary implant stability. Lekholm and Zarb [1] suggested a bone classification system based on macrostructure, where the morphology and distribution of cortical and trabecular bones determine bone quality. However, Lindh et al. [2] proposed a new classification system using reference images to assess the trabecular pattern in periapical radiographs in the mandible before implant treatment, because the accuracy of the classification system of Lekholm and Zarb could not be evaluated. Following the introduction of multislice computed tomography (CT) for preoperative evaluation of bone density in Hounsfield units (HU) as a parameter of bone quality, Norton and Gamble [3] proposed a new classification system based on bone HU and related this new classification to that of Lekholm and Zarb [1]. Recently, the use of cone-beam (CB) CT in dentistry has increased, because CBCT is associated with benefits such as increased patient comfort, lower radiation doses, and lower operation costs compared to conventional CT [4]. However, Nackaerts et al. [5] demonstrated that density profiles of conventional CT showed stable HU values whereas intensity values in CBCT images are not reliable because the values are influenced by the device used, imaging parameters, and positioning. Accordingly, Naitoh et al. [6] found that the trabecular bone volume per total tissue volume obtained using CBCT images was closely correlated with $\mathrm{HU}$ values generated from conventional CT images.

Primary implant stability has been acknowledged as an essential criterion for later achievement of osseointegration [7]. Primary stability is assessed by resonance frequency analysis using the Osstell device, for which damping capacity assessment is considered reliable, reproducible, and easy to use [8]. Veltri et al. [9] demonstrated that, for optimal implant stability, damping values should be in the range of satisfactory values proposed in the literature, although these values are not related to the radiographic trabecular bone pattern. Lachmann et al. [10] suggested that the Osstell device may be useful for monitoring the state of an individual implant over time, because the outcome of implant stability 
TABLE 1: Bone densities in Hounsfield units (HU) for males and females.

\begin{tabular}{lccccc}
\hline & & & \multicolumn{2}{c}{ Bone densities (HU) } \\
Gender & Number of implant sites & Age, years (SD) & Inside (SD) & Outside (SD) & Combination of inside and outside (SD) \\
\hline Male & 37 & $55.8(12.3)$ & $700.2(185.2)^{\mathrm{a}}$ & $751.1(236.4)$ & $725.7(174.7)^{\mathrm{c}}$ \\
Female & 67 & $57.1(10.7)$ & $474.2(230.4)^{\mathrm{a}, \mathrm{b}}$ & $619.6(208.8)^{\mathrm{b}}$ & $546.9(209.7)^{\mathrm{c}}$ \\
\hline
\end{tabular}

The presence of the same superscript letter indicates a significant difference (all cases: $P<0.01$ ).

depends on environmental factors such as bone quality and implant geometry. Merheb et al. [7] showed that a significant linear relationship exists between damping values and $\mathrm{HU}$ values at implant insertion and suggested that preoperative evaluation of cortical thickness and trabecular bone $\mathrm{HU}$ appears to be the most reliable method for predicting implant stability.

The purpose of this study was to evaluate preoperative CT-derived bone densities in $\mathrm{HU}$ and their association with acquired primary implant stability, and to compare these values to the optimal bone densities proposed in the literature.

\section{Materials and Methods}

2.1. Patients and Implants. Fifty-one patients (18 males, average age $55.8 \pm 12.3$ years, range $19-76$; 33 females, average age $57.1 \pm 10.7$ years, range $29-77)$ who had undergone implant placement between 2003 and 2010 using the Strauman Dental Implant System (Basel, Switzerland) or GC Implant Re System Genesio (Tokyo, Japan) were selected for this study (Table 1). None of the patients selected suffered from a systemic disease. Implants were placed via a surgical template for optimal localization and optimal acquired primary stability. This study was approved by the ethics committee of Hiroshima University Hospital (no. 214).

2.2. Bone Density. To evaluate the bone density of each patient before implant planning, a CT scanner (Aquilion TSX-101A; Toshiba Medical Systems, Otawara, Japan) with the following technical parameters was used: $0.5 \mathrm{~s}, 0.5 \mathrm{~mm}$ slice thickness, $0.3 \mathrm{~mm}$ slice increment, $135 \mathrm{kV}, 150 \mathrm{~mA}$, and $0^{\circ}$ gantry angulation. CT images were stored in DICOM format. The images were loaded onto SimPlant software (Materialise Dental Japan, Tokyo, Japan), which enables the construction of a 3 dimensional model of each maxilla or mandible and the determination of bone densities to a thickness of $1 \mathrm{~mm}$ inside and $1 \mathrm{~mm}$ outside the simulated implant. Bone density was measured in HU. Software-based analysis of bone densities was performed by 1 operator determining the position of implant in the optimum size, type, and angulation. The data were analyzed using the Kruskal-Wallis test and Scheffe's test for multiple comparisons $(P<0.05)$.

\section{Results}

The study cohort included 37 implant sites in male participants and 67 implant sites in female participants; the mean bone density $(725.7 \pm 174.7 \mathrm{HU})$ in males was significantly higher than that $(546.9 \pm 209.7 \mathrm{HU})$ in females $(P<$ 0.01 ; Table 1). In females, the mean bone density (474.2 \pm $230.4 \mathrm{HU}$ ) inside the implant was significantly lower than that $(619.6 \pm 208.8)$ outside the implant $(P<0.01)$.

The bone densities in HU for different jaw sections (maxilla and mandible), regions (anterior and posterior), and operating procedures (1- and 2-stage) are shown in Table 2. The mean bone density $(540.9 \pm 201.9 \mathrm{HU})$ in the maxilla (31 implant sites) was significantly lower than that $(640.0 \pm 214.9)$ in the mandible (73 implant sites; $P<0.05)$. The mean bone density $(695.0 \pm 206.0 \mathrm{HU})$ in the anterior region (15 implant sites) was higher than that (596.2 \pm $214.3 \mathrm{HU}$ ) in the posterior region (89 implant sites), but this difference was not significant $(P>0.05)$. The mean bone density $(630.8 \pm 223.1 \mathrm{HU})$ in patients who had undergone the 1-stage procedure (66 implant sites) was higher than that $(575.2 \pm 198.1 \mathrm{HU})$ in patients who had undergone the 2stage procedure (38 implant sites), but this difference was not significant $(P>0.05)$.

The mean bone density was highest in the anterior mandible $(843.6 \pm 241.9 \mathrm{HU})$, followed by the anterior maxilla $(640.9 \pm 172.7 \mathrm{HU})$, the posterior mandible $(628.2 \pm$ $209.2 \mathrm{HU})$, and the posterior maxilla $(486.0 \pm 199.2 \mathrm{HU}$; Table 3). There were significant differences among 4 jaw regions $(P<0.05)$, but the difference between each region was not significant $(P>0.05)$.

\section{Discussion}

In this study, which assessed preoperative CT-derived bone densities in $\mathrm{HU}$, the mean bone density in males was significantly higher than that in females, which is similar to findings reported by Turkyilmaz et al. [11]. Likewise, the tendency for the mean bone densities of the 4 jaw regions to decrease, as seen in this study, in the order of anterior mandible, anterior maxilla, posterior mandible, and posterior maxilla is similar to that observed in previous studies [3, 11-15].

A summary of the previously described bone density classification systems $[1,3,12,16-18]$ is shown in Table 3. In the Lekholm and Zarb classification [1], bone density is graded as follows: Q1, almost the entire jaw is comprised of homogenous compact bone; Q2, a thick layer of compact bone surrounds a core of dense trabecular bone; Q3, a thin layer of cortical bone surrounds a core of dense trabecular bone; and Q4, a thin layer of cortical bone surrounds a core of low-density trabecular bone. Bone quality scoring in the rage of Q2 and Q3 is associated with good prospects for implant success, easier implant placement, better primary fixation, and the use of standard instruments and 
TABLE 2: Bone densities in Hounsfield units (HU) for different jaw sections, regions, and operating procedures.

\begin{tabular}{|c|c|c|c|c|}
\hline & \multirow[b]{2}{*}{ Number of implant sites } & \multicolumn{3}{|c|}{ Bone densities (HU) } \\
\hline & & Inside (SD) & Outside (SD) & Combination of inside and outside (SD) \\
\hline \multicolumn{5}{|l|}{ Jaw section } \\
\hline Maxilla & 31 & $523.8(267.5)$ & $558.1(202.2)^{\mathrm{a}}$ & $540.9(201.9)^{\mathrm{b}}$ \\
\hline Mandible & 73 & $567.6(228.7)$ & $712.4(222.3)^{\mathrm{a}}$ & $640.0(214.9)^{\mathrm{b}}$ \\
\hline \multicolumn{5}{|l|}{ Region } \\
\hline Anterior & 15 & $659.6(270.1)$ & $730.4(279.1)$ & $695.0(206.0)$ \\
\hline Posterior & 89 & $536.9(232.1)$ & $655.6(216.9)$ & $596.2(214.3)$ \\
\hline \multicolumn{5}{|l|}{ Procedure } \\
\hline 1-stage & 66 & $571.5(233.8)$ & $690.1(235.6)$ & $630.8(223.1)$ \\
\hline 2-stage & 38 & $525.1(251.9)$ & $625.2(207.6)$ & $575.2(198.1)$ \\
\hline
\end{tabular}

The presence of the same superscript letter indicates a significant difference $\left({ }^{\mathrm{a}} P<0.01 ;{ }^{\mathrm{b}} P<0.05\right)$.

TABLE 3: Bone classifications and bone densities in Hounsfield units (HU) observed in this study and previous studies for different jaw regions.

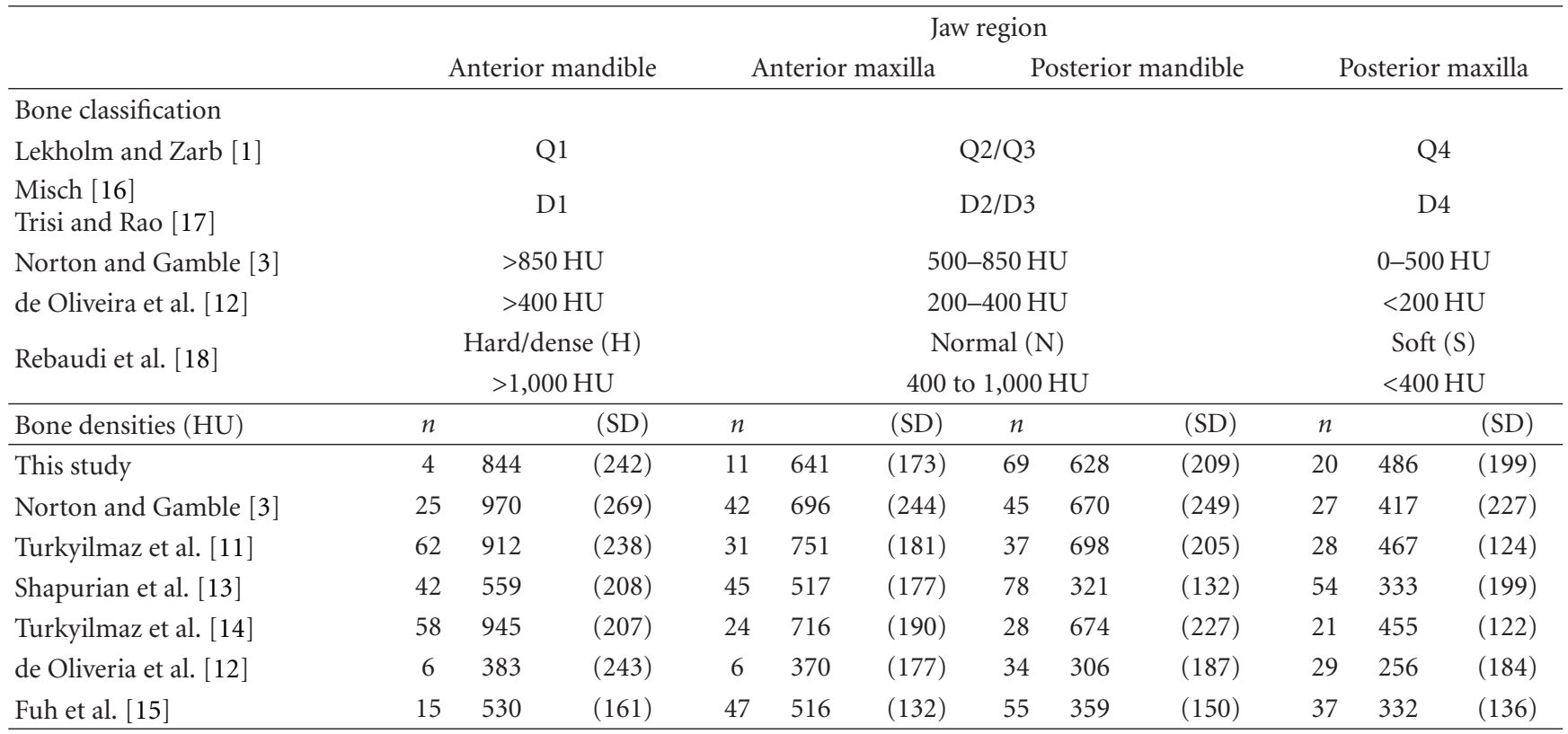

Lekholm and Zarb classification based on bone macrostructure: Q1, almost the entire jaw is comprised of homogenous compact bone; Q2, a thick layer of compact bone surrounds a core of dense trabecular bone; Q3, a thin layer of cortical bone surrounds a core of dense trabecular bone; Q4, a thin layer of cortical bone surrounds a core of low-density trabecular bone. Misch classification based on the clinical drilling resistance of the bone: D1, oak or maple wood; D2, white pine of spruce wood; D3, balsa wood; D4, styrofoam. $n$ : number of implant sites.

components. Misch [16] defined 4 bone density classes (D1, $\mathrm{D} 2, \mathrm{D} 3$, and D4) based on the clinical drilling resistance of the bone. Based on the Misch classification [16], Trisi and Rao [17] attempted to establish a quantitative threshold of bone volume (\%) for the 4 classes and suggested combining D2 and D3 into 1 group, thus classifying bone density into 3 groups of clinical interest: D1, D2/D3, and D4. For bone quality assessment in accordance with the Lekholm and Zarb classification [1], Norton and Gamble [3] found a strong correlation between $\mathrm{HU}$ values and sites classified as Q1 and Q4, and thus proposed unifying Q2 and Q3. In addition, they applied the bone density range in HU to the Lekholm and Zarb classification [1]. The CT software used in this study was the same as that used for all previous bone density classifications, and most of the bone densities observed for the different jaw regions in this study were in the range of corresponding optimal bone densities described in these classification systems. Because the bone density range in $\mathrm{HU}$ proposed by de Oliveira et al. [12] focused on the trabecular bone only, the bone densities observed in the present study were higher, as the latter included both the cortical and trabecular bone. Recently, Rebaudi et al. [18] introduced a novel bone quality/density classification system that divides bone into 3 classes: hard/dense $(\mathrm{H})$, corresponding to $\mathrm{Q} 1$ and $\mathrm{D} 1$; normal $(\mathrm{N})$, corresponding to Q2/Q3 and D2/D3; soft (S), corresponding to Q4 and D4 (HNS classification). Hard bone $(>1,000 \mathrm{HU})$ and soft bone $(<400 \mathrm{HU})$ are associated with a higher risk of implant failure whereas normal bone 
(400-1,000 HU) represents a safe zone. In this study, the optimal bone density range for primary implant stability was set at 400-1,000 HU, corresponding to the classification of normal bone proposed by Rebaudi et al. [18], which indeed seems to lead to implant success. Furthermore, the mean bone density in patients who underwent the 1stage procedure was justifiably higher than that in patients who underwent the 2-stage procedure, as the former bone densities belonged to the category of normal bone.

Stoppie et al. [19] demonstrated that predictions of the mechanical properties of the trabecular bone are only valid for implant sites fully situated in the trabecular bone or with a very small amount of cortical bone involvement. This observation indicates that bone densities assessed by $\mathrm{HU}$, as performed in the current study, may be more accurate for the prediction of primary implant stability, except in jaws with a thicker cortical layer. Indeed, Farré-Pagès et al. [20] concluded that $\mathrm{HU}$ can be used as a diagnostic parameter to predict possible implant stability. Thus, preoperative assessment of bone densities by HU is very important for optimizing primary implant stability.

\section{Conclusions}

In this study, the mean HU bone density in the 4 jaw regions decreased in the following order: anterior mandible, anterior maxilla, posterior mandible, and posterior maxilla, which is similar to reported reference values. Furthermore, the mean bone densities predicted to maximize acquired primary implant stability, as assessed by HU, were in the optimal range proposed in the literature. CT using $\mathrm{HU}$ is therefore a suitable assessment tool for bone densities prior to dental implantation.

\section{Acknowledgments}

This paper was partly supported by a Grant-in-Aid for Scientific Research (no. 23592887) from the Japan Society for the Promotion of Science (JSPS) and the Ministry of Education, Culture, Sports, Science, and Technology (MEXT), Japan (2011-2013). The authors would like to thank Editage for providing editorial assistance.

\section{References}

[1] U. Lekholm and G. A. Zarb, "Patient selection and preparation," in Tissue Integrated Prostheses: Osseointegration in Clinical Dentistry, P. I. Brånemark, G. A. Zarb, and T. Alberktsson, Eds., pp. 199-209, Quintessence, Chicago, Ill, USA, 1985.

[2] C. Lindh, A. Petersson, and M. Rohlin, "Assessment of the trabecular pattern before endosseous implant treatment Diagnostic outcome of periapical radiography in the mandible," Oral Surgery, Oral Medicine, Oral Pathology, Oral Radiology, and Endodontics, vol. 82, no. 3, pp. 335-343, 1996.

[3] M. R. Norton and C. Gamble, "Bone classification: an objective scale of bone density using the computerized tomography scan," Clinical Oral Implants Research, vol. 12, no. 1, pp. 79-84, 2001.
[4] W. C. Scarfe, A. G. Farman, and P. Sukovic, "Clinical applications of cone-beam computed tomography in dental practice," Journal of the Canadian Dental Association, vol. 72, no. 1, pp. 75-80, 2006.

[5] O. Nackaerts, F. Maes, H. Yan, P. Couto Souza, R. Pauwels, and R. Jacobs, "Analysis of intensity variability in multislice and cone beam computed tomography," Clinical Oral Implants Research, vol. 22, pp. 873-879, 2011.

[6] M. Naitoh, H. Aimiya, A. Hirukawa, and E. Ariji, "Morphometric analysis of mandibular trabecular bone using cone beam computed tomography: an in vitro study," International Journal of Oral and Maxillofacial Implants, vol. 25, pp. 10931098, 2010.

[7] J. Merheb, N. Van Assche, W. Coucke, R. Jacobs, I. Naert, and M. Quirynen, "Relationship between cortical bone thickness or computerized tomography-derived bone density values and implant stability," Clinical Oral Implants Research, vol. 21, no. 6, pp. 612-617, 2010.

[8] R. Nedir, M. Bischof, S. Szmukler-Moncler, J. P. Bernard, and J. Samson, "Predicting osseointegration by means of implant primary stability: a resonance-frequency analysis study with delayed and immediately loaded ITI SLA implants," Clinical Oral Implants Research, vol. 15, no. 5, pp. 520-528, 2004.

[9] M. Veltri, P. Balleri, and M. Ferrari, "Damping factor for monitoring the bone interface at dental implants," Clinical Oral Implants Research, vol. 18, no. 6, pp. 738-742, 2007.

[10] S. Lachmann, J. Y. Laval, D. Axmann, and H. Weber, "Influence of implant geometry on primary insertion stability and simulated peri-implant bone loss: an in vitro study using resonance frequency analysis and damping capacity assessment," International Journal of Oral and Maxillofacial Implants, vol. 26, pp. 347-355, 2011.

[11] I. Turkyilmaz, T. F. Tözüm, C. Tumer, and E. N. Ozbek, "Assessment of correlation between computerized tomography values of the bone, and maximum torque and resonance frequency values at dental implant placement," Journal of Oral Rehabilitation, vol. 33, no. 12, pp. 881-888, 2006.

[12] R. C. de Oliveira, C. R. Leles, L. M. Normanha, C. Lindh, and R. F. Ribeiro-Rotta, "Assessments of trabecular bone density at implant sites on CT images," Oral Surgery, Oral Medicine, Oral Pathology, Oral Radiology and Endodontology, vol. 105, no. 2, pp. 231-238, 2008.

[13] T. Shapurian, P. D. Damoulis, G. M. Reiser, T. J. Griffin, and W. M. Rand, "Quantitative evaluation of bone density using the Hounsfield index," International Journal of Oral and Maxillofacial Implants, vol. 21, no. 2, pp. 290-297, 2006.

[14] I. Turkyilmaz, T. F. Tözüm, and C. Tumer, "Bone density assessments of oral implant sites using computerized tomography," Journal of Oral Rehabilitation, vol. 34, no. 4, pp. 267272, 2007.

[15] L. J. Fuh, H. L. Huang, C. S. Chen et al., "Variations in bone density at dental implant sites in different regions of the jawbone," Journal of Oral Rehabilitation, vol. 37, no. 5, pp. 346-351, 2010.

[16] C. E. Misch, "Density of bone: effect on treatment plans, surgical approach, healing, and progressive boen loading," International Journal of Oral Implantology, vol. 6, no. 2, pp. 23-31, 1990.

[17] P. Trisi and W. Rao, "Bone classification: clinicalhistomorphometric comparison," Clinical Oral Implants Research, vol. 10, no. 1, pp. 1-7, 1999.

[18] A. Rebaudi, P. Trisi, R. Cella, and G. Cecchini, "Preoperative evaluation of bone quality and bone density using a novel CT/microCT-based hard-normal-soft classification system," 
International Journal of Oral and Maxillofacial Implants, vol. 25, no. 1, pp. 75-85, 2010.

[19] N. Stoppie, V. Pattijn, T. Van Cleynenbreugel, M. Wevers, J. V. Sloten, and N. Ignace, "Structural and radiological parameters for the characterization of jawbone," Clinical Oral Implants Research, vol. 17, no. 2, pp. 124-133, 2006.

[20] N. Farré-Pagès, Ma. L. Augé-Castro, F. Alaejos-Algarra, J. Mareque-Bueno, E. Ferrés-Padró, and F. Hernández-Alfaro, "Relation between bone density and primary implant stability," Medicina Oral, Patologia Oral y Cirugia Bucal, vol. 16, no. 1, pp. e62-e67, 2011. 


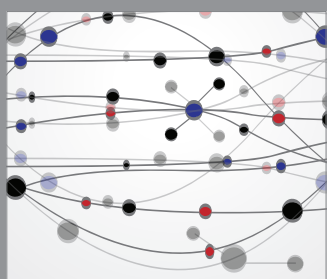

The Scientific World Journal
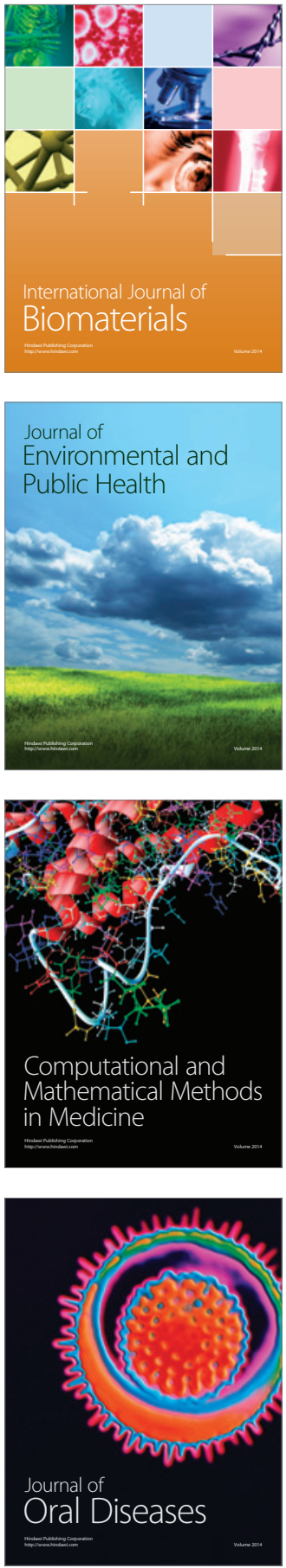
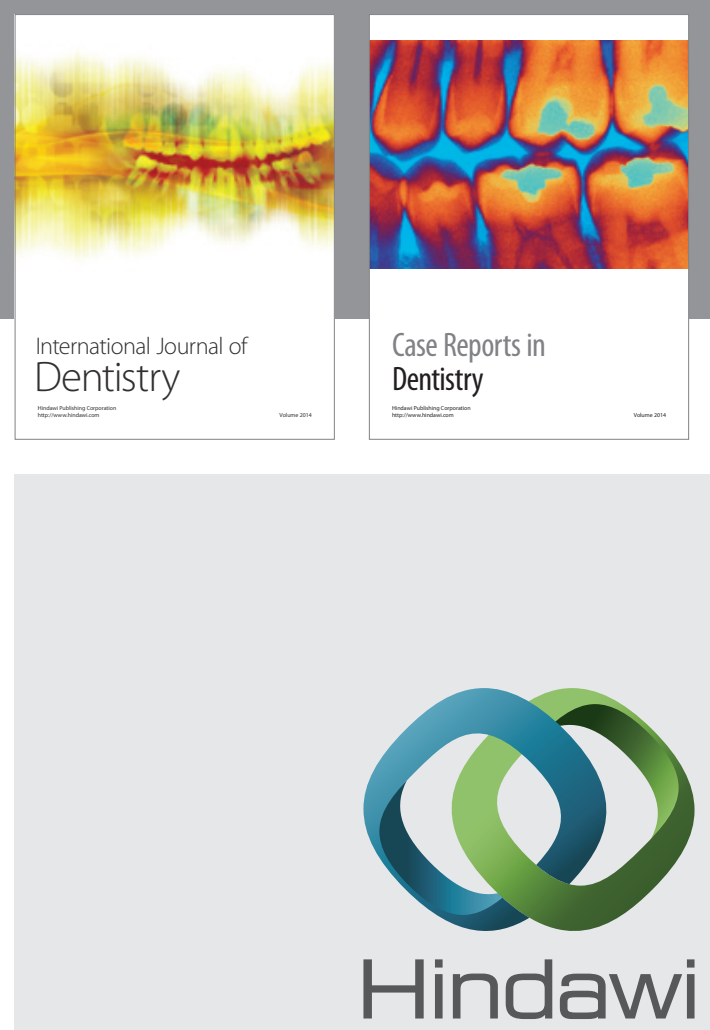

Submit your manuscripts at

http://www.hindawi.com
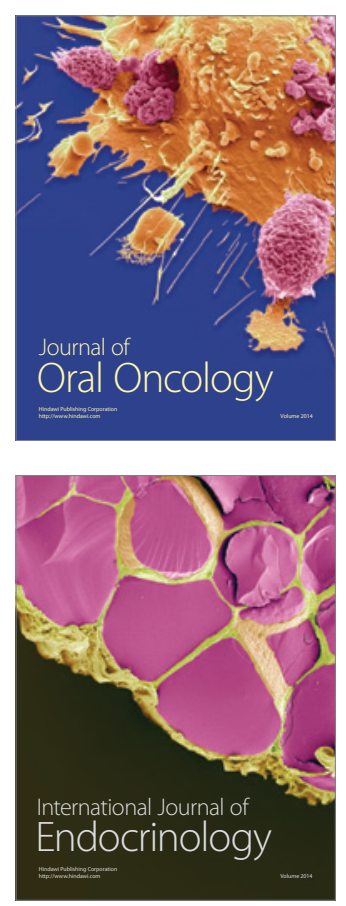
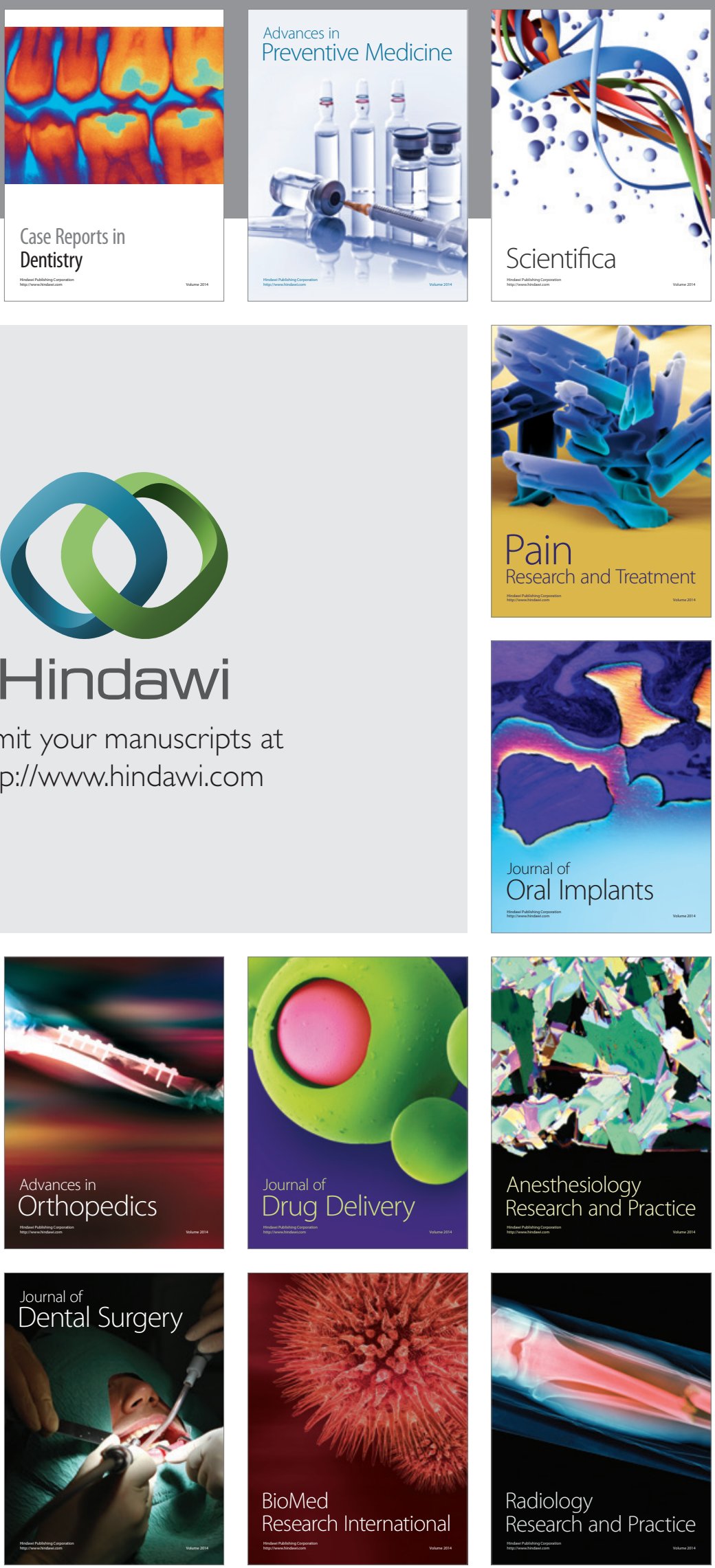